\title{
Some results on higher orders quasi-isometries
}

\author{
Sid Ahmed Ould Ahmed Mahmoud*1 (D), Adel Saddi² (D), Khadija Gherairi² (D) \\ ${ }^{1}$ Mathematics Department, College of Science, Jouf University, Sakaka P.O.Box 2014. Saudi Arabia \\ ${ }^{2}$ Laboratory of Mathematics and Applications LR17ES11, College of Science, Gabes University, Tunisia
}

\begin{abstract}
The purpose of the present paper is to pursue further study of a class of linear bounded operators, known as $n$-quasi- $m$-isometric operators acting on an infinite complex separable Hilbert space $\mathcal{H}$. We give an equivalent condition for any $T$ to be $n$-quasi- $m$-isometric operator. Using this result we prove that any power of an $n$-quasi- $m$-isometric operator is also an $n$-quasi- $m$-isometric operator. In general the converse is not true. However, we prove that if $T^{r}$ and $T^{r+1}$ are $n$-quasi- $m$-isometries for a positive integer $r$, then $\mathrm{T}$ is an $n$-quasi- $m$-isometric operator. We study the sum of an $n$-quasi- $m$-isometric operator with a nilpotent operator. We also study the product and tensor product of two $n$-quasi- $m$ isometries. Further, we define $n$-quasi strict $m$-isometric operators and prove their basic properties.
\end{abstract}

Mathematics Subject Classification (2010). 47B99, 47A05

Keywords. $m$-isometry, strict $m$-isometry, $n$-quasi- $m$-isometry

\section{Introduction}

Throughout this paper, $\mathbb{N}$ denotes the set of non negative integers, $\mathcal{H}$ stands for an infinite separable complex Hilbert space with inner product $\langle. \mid\rangle,. \mathcal{L}(\mathcal{H})$ is the Banach algebra of all bounded linear operators on $\mathcal{H}$ and $I=I_{\mathcal{H}}$ the identity operator. For every $T \in \mathcal{L}(\mathcal{H})$ we denote by $\mathcal{R}(T), \mathcal{N}(T)$ and $T^{*}$ the range, the null space and the adjoint of $T$, respectively. A closed subspace $\mathcal{M} \subset \mathcal{H}$ is invariant for $T$ (or $T$-invariant) if $T \mathcal{M} \subset \mathcal{M}$. As usual, the orthogonal complement and the closure of $\mathcal{M}$ are denoted $\mathcal{M}^{\perp}$ and $\overline{\mathcal{M}}$, respectively. We denote by $P_{\mathcal{M}}$ the orthogonal projection on $\mathcal{M}$.

Some of the most important subclasses of the algebra of all bounded linear operators acting on a Hilbert space, are the classes of partial isometries and quasi-isometries. An operator $T \in \mathcal{L}(\mathcal{H})$ is said to be an isometry if $T^{*} T=I$, a partial isometry if $T T^{*} T=T$ and quasi-isometry if $T^{* 2} T^{2}=T^{*} T$.

In recent years these classes has been generalized, in some sense, to the larger sets of operators so-called $m$-isometries, $m$-partial isometries and $n$-quasi-isometries. An operator $T \in \mathcal{L}(\mathcal{H})$ is said to be

\footnotetext{
*Corresponding Author.

Email addresses: sidahmed@ju.edu.sa; sidahmed.sidha@gmail.com (S.A.O.A. Mahmoud), saddi.adel@gmail.com (A. Saddi), khadija.gherairi@gmail.com (K. Gherairi)

Received: 27.02.2019; Accepted: 12.09.2019
} 
(1) $m$-isometric operator for some integer $m \geq 1$ if it satisfies the operator equation

$$
\sum_{0 \leq k \leq m}(-1)^{m-k}\left(\begin{array}{c}
m \\
k
\end{array}\right) T^{* k} T^{k}=0,
$$

It is immediate that $T$ is $m$-isometric operator if and only if

$$
\sum_{0 \leq k \leq m}(-1)^{m-k}\left(\begin{array}{c}
m \\
k
\end{array}\right)\left\|T^{k} x\right\|^{2}=0 \quad \forall x \in \mathcal{H} .
$$

(2) $m$-partial-isometry for some integer $m \geq 1$ if

$$
T\left(\sum_{0 \leq k \leq m}(-1)^{k}\left(\begin{array}{c}
m \\
k
\end{array}\right) T^{* m-k} T^{m-k}\right)=0,
$$

(3) $(m, q)$-partial isometry (or $q$-partial- $m$-isometry) if

$$
T^{q}\left(\sum_{0 \leq k \leq m}(-1)^{k}\left(\begin{array}{c}
m \\
k
\end{array}\right) T^{* m-k} T^{m-k}\right)=0,
$$

(4) $m$-quasi-isometry for some integer $n \geq 1$ if

$$
T^{* m+1} T^{m+1}-T^{* m} T^{m}=0 .
$$

It is immediate that $T$ is $m$-quasi-isometric if and only if

$$
T^{* m}\left(T^{*} T-I\right) T^{m}=0 .
$$

Here $\left(\begin{array}{c}m \\ k\end{array}\right)$ is the binomial coefficient. In [1], J. Agler and M. Stankus initiated the study of operators $T$ that satisfy the identity (1.1). In [24], A. Saddi and O. A. M. Sid Ahmed studied an operator $T$ which satisfies (1.3). This concept was later generalized to the operators satisfying (1.4), was defined by O. A. M. Sid Ahmed [17]. The study of operators satisfying (1.5) was introduced and study by L. Suciu in [25]. The 1-quasi-isometries are shortly called quasi-isometries, such operators being firstly studied in [20] and [22].

Recently, S. Mecheri and T. Prasad [19] introduced the class of $n$-quasi-m-isometric operators which generalizes the class of $m$-isometric operators and $n$-quasi-isometries. For positive integers $m$ and $n$, an operator $T \in \mathcal{L}(\mathcal{H})$ is said to be an $n$-quasi- $m$-isometric operator if

$$
T^{* n}\left(\sum_{0 \leq k \leq m}(-1)^{k}\left(\begin{array}{c}
m \\
k
\end{array}\right) T^{* m-k} T^{m-k}\right) T^{n}=0,
$$

After an introduction on the subject and some connection with known facts in this context, the results of the paper are briefly described. In section two, we give a matrix characterization of $n$-quasi- $m$-isometries by using the decomposition $\mathcal{H}=\overline{T^{n}(\mathcal{H})} \oplus T^{*-n}(0)$. Several properties are proved by exploiting the special kind of operator matrix representation associated with such operators. In the course of our investigation, we find some properties of $m$-isometries which are retained by $n$-quasi- $m$-isometries. In particular, we show that if $T \in \mathcal{L}(\mathcal{H})$ is an $n$-quasi-isometry then its power is an $n$-quasi-isometry. If $T$ and $S$ are doubly commuting such that $T$ is an $n_{1}$-quasi-m-isometry and $S$ is an $n_{2}$-quasi- $l$-isometry, then $T S$ is a $n_{0}=\max \left\{n_{1}, n_{2}\right\}$-quasi- $(m+l-1)$-isometry. It has also been proved that the sum of an $n$-quasi- $m$-isometry and a commuting nilpotent operator of degree $p$ is a $2 \max \{n, p\}$-quasi- $(m+2 p-2)$-isometry. In section three, we recall the definition of $n$ quasi strict- $m$-isometries and we give some of their properties which are similar to those of $n$-quasi- $m$-isometries. 


\section{Some properties of $n$-quasi-m-isometric operators}

In this section, we study some further properties of $n$-quasi-m-isometries. First, we will start with the following notations.

For $T \in \mathcal{L}(\mathcal{H})$, we set

$$
\begin{gathered}
\beta_{m}(T):=\sum_{0 \leq k \leq m}(-1)^{m-k}\left(\begin{array}{c}
m \\
k
\end{array}\right) T^{* k} T^{k} \\
\beta_{m, n}(T):=T^{* n}\left(\sum_{0 \leq k \leq m}(-1)^{m-k}\left(\begin{array}{c}
m \\
k
\end{array}\right) T^{* k} T^{k}\right) T^{n}
\end{gathered}
$$

and

$$
\Delta_{m, n}(T, x):=\sum_{0 \leq k \leq m}(-1)^{m-k}\left(\begin{array}{c}
m \\
k
\end{array}\right)\left\|T^{k+n} x\right\|^{2}, \quad x \in \mathcal{H} .
$$

Observe that $T$ is an $n$-quasi- $m$-isometric operator if and only if $\beta_{m, n}(T)=0$ or equivalently, if

$$
\Delta_{m, n}(T, x)=0, \quad \forall x \in \mathcal{H}
$$

Lemma 2.1. Let $T \in \mathcal{L}(\mathcal{H})$. Then $T$ is an $n$-quasi-m-isometric operator if and only if

$$
\sum_{0 \leq k \leq m}(-1)^{m-k}\left(\begin{array}{c}
m \\
k
\end{array}\right)\left\|T^{k} x\right\|^{2}=0 \quad \forall x \in \overline{\mathcal{R}\left(T^{n}\right)},
$$

i.e., $T$ is an m-isometric operator on $\overline{\mathcal{R}\left(T^{n}\right)}$.

Proof. The proof is obvious.

Let $\mathbb{Z}$ denote the set of integers and $\mathbb{Z}_{+}$denote the set of nonnegative integers.

Lemma 2.2 ([13, Lemma 5.4]). Let $\left(a_{j}\right)_{j \in \mathbb{Z}_{+}}$be a sequence of real numbers. Then

$$
\sum_{0 \leq k \leq m}(-1)^{m-k}\left(\begin{array}{c}
m \\
k
\end{array}\right) a_{j+k}=0 \text { for } j \geq 0
$$

if and only if there exists a polynomial $P$ of degree less than or equal to $m-1$ such that $a_{j}=P(j)$. In this case $P$ is the unique polynomial interpolating $\left\{\left(j, a_{j}\right)\right\}, 0 \leq j \leq m-1$.

Proposition 2.3. Let $T \in \mathcal{L}(\mathcal{H})$ and $x \in \mathcal{H}$. Then $T$ is an n-quasi-m-isometry if and only if for each $x \in \mathcal{H}$, there exists a polynomial $P$ of degree less than or equal to $m-1$, such that $P(j)=\left\|T^{n+j} x\right\|^{2}$ for $j \in \mathbb{Z}_{+}$.

Proof. The proof is a consequence of Lemma 2.1, Lemma 2.2 and [13, Theorem 5.5].

In [19], S. Mecheri and T. Prasad studied the matrix representation of $n$-quasi-misometric operator with respect to the direct sum of $\overline{\mathcal{R}\left(T^{n}\right)}$ and its orthogonal complement. In the following, we give an equivalent condition for $T$ to be $n$-quasi- $m$-isometric operator. Using this result we obtained several important properties of this class of operators.

Theorem 2.4. Let $T \in \mathcal{L}(\mathcal{H})$ such that $\overline{\mathcal{R}\left(T^{n}\right)} \neq \mathcal{H}$, then the following statements are equivalent.

(1) $T$ is an n-quasi-m-isometric operator.

(2) $T=\left(\begin{array}{cc}T_{1} & T_{2} \\ 0 & T_{3}\end{array}\right)$ on $\mathcal{H}=\overline{\mathcal{R}\left(T^{n}\right)} \oplus \mathcal{N}\left(T^{* n}\right)$, where $T_{1}$ is an m-isometric operator and $T_{3}^{n}=0$. 
Proof. (1) $\Rightarrow$ (2) It follows from [19, Lemma 2.1].

(2) $\Rightarrow$ (1) Suppose that $T=\left(\begin{array}{cc}T_{1} & T_{2} \\ 0 & T_{3}\end{array}\right)$ on $\mathcal{H}=\overline{\mathcal{R}\left(T^{n}\right)} \oplus \mathcal{N}\left(T^{* n}\right)$, with

$$
\beta_{m}\left(T_{1}\right)=0 \text { and } T_{3}^{n}=0 .
$$

Since $T^{k}=\left(\begin{array}{cc}T_{1}^{k} & \sum_{0 \leq j \leq k-1} T_{1}^{j} T_{2} T_{3}^{k-1-j} \\ 0 & T_{3}^{k}\end{array}\right)$ for all $k \geq 1$, we have

$$
\begin{aligned}
& \beta_{m, n}(T)=T^{* n}\left(\sum_{0 \leq k \leq m}(-1)^{m-k}\left(\begin{array}{c}
m \\
k
\end{array}\right) T^{* k} T^{k}\right) T^{n} \\
& =\left(\begin{array}{cc}
T_{1} & T_{2} \\
0 & T_{3}
\end{array}\right)^{* n}\left\{(-1)^{m} I+\sum_{1 \leq k \leq m}(-1)^{m-k}\left(\begin{array}{c}
m \\
k
\end{array}\right)\left(\begin{array}{cc}
T_{1} & T_{2} \\
0 & T_{3}
\end{array}\right)^{* k}\left(\begin{array}{cc}
T_{1} & T_{2} \\
0 & T_{3}
\end{array}\right)^{k}\right\} \\
& \times\left(\begin{array}{cc}
T_{1} & T_{2} \\
0 & T_{3}
\end{array}\right)^{n}
\end{aligned}
$$

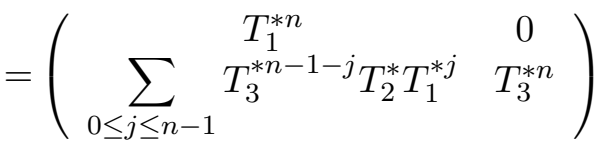

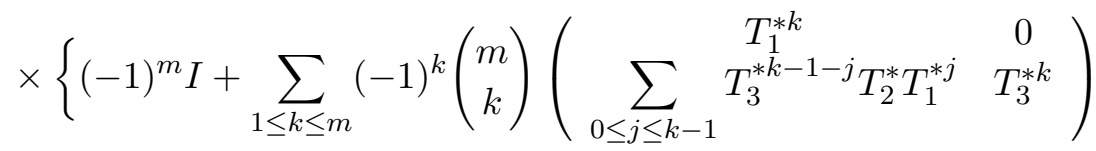

$$
\begin{aligned}
& \left.\times\left(\begin{array}{cc}
T_{1}^{k} & \sum_{j=0}^{k-1} T_{1}^{j} T_{2} T_{3}^{k-1-j} \\
0 & T_{3}^{k}
\end{array}\right)\right\} \\
& \times\left(\begin{array}{cc}
T_{1}^{n} & \sum_{0 \leq j \leq n-1} T_{1}^{j} T_{2} T_{3}^{n-1-j} \\
0 & T_{3}^{n}
\end{array}\right)
\end{aligned}
$$

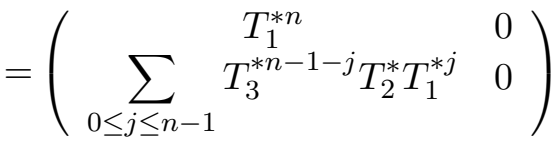

$$
\begin{aligned}
& \times\left\{\left(\begin{array}{cc}
\beta_{m}\left(T_{1}\right) & C \\
D & B
\end{array}\right)\right\} \times\left(\begin{array}{cc}
T_{1}^{n} & \sum_{0 \leq j \leq n-1} T_{1}^{j} T_{2} T_{3}^{n-1-j} \\
0 & 0
\end{array}\right)
\end{aligned}
$$

where $B, C, D \in \mathcal{L}(\mathcal{H})$. Moreover

$$
\begin{aligned}
& \beta_{m, n}(T) \\
& =\left(\begin{array}{cc}
T_{1}^{* n} \beta_{m}\left(T_{1}\right) T_{1}^{n} & T_{1}^{* n} \beta_{m}\left(T_{1}\right) \sum_{0 \leq j \leq n-1} T_{1}^{j} T_{2} T_{3}^{n-1-j} \\
\sum_{0 \leq j \leq n-1} T_{3}^{* n-1-j} T_{2}^{*} T_{1}^{* j} \beta_{m}\left(T_{1}\right) T_{1}^{n} & \sum_{0 \leq j \leq n-1} T_{3}^{* n-1-j} T_{2}^{*} T_{1}^{* j} \beta_{m}\left(T_{1}\right) \sum_{0 \leq j \leq n-1} T_{1}^{j} T_{2} T_{3}^{n-1-j}
\end{array}\right) .
\end{aligned}
$$

Since $\beta_{m}\left(T_{1}\right)=0$, it follows that $\beta_{m, n}(T)=0$. Thus $T$ is an $n$-quasi- $m$-isometric operator.

For $T \in \mathcal{L}(\mathcal{H})$, we denote by $\sigma(T), \sigma_{a p}(T)$ and $\sigma_{p}(T)$ the spectrum, the approximate point spectrum and the point spectrum of $T$, respectively. 
Corollary 2.5. Let $T \in \mathcal{L}(\mathcal{H})$ be an n-quasi-m-isometric operator. The following statements hold.

(i) $\sigma(T)=\sigma\left(T_{1}\right) \cup\{0\}$ where $T_{1}=\left.T\right|_{\overline{\mathcal{R}\left(T^{n}\right)}}$.

(ii) $T_{1}$ is bounded below.

(iii) If $\mu \in \sigma_{a p}(T) \backslash\{0\}$ then $\bar{\mu} \in \sigma_{a p}\left(T^{*}\right)$. In particular, if $\mu \in \sigma_{p}(T) \backslash\{0\}$ then $\bar{\mu} \in \sigma_{p}\left(T^{*}\right)$.

Proof. (i) Since $T$ is an $n$-quasi- $m$-isometric operator, it follows from Theorem 2.4 that

$$
T=\left(\begin{array}{cc}
T_{1} & T_{2} \\
0 & T_{3}
\end{array}\right) \text { on } \mathcal{H}=\overline{\mathcal{R}\left(T^{n}\right)} \oplus \mathcal{N}\left(T^{* n}\right),
$$

where $T_{1}$ is an $m$-isometric operator and $T_{3}^{n}=0$. From [15, Corollary 7], it follows that $\sigma(T) \cup W=\sigma\left(T_{1}\right) \cup \sigma\left(T_{3}\right)$, where $W$ is the union of certain of the holes in $\sigma(T)$ which is a subset of $\sigma\left(T_{1}\right) \cap \sigma\left(T_{3}\right)$. Further $\sigma\left(T_{3}\right)=\{0\}$ and $\sigma\left(T_{1}\right) \cap \sigma\left(T_{3}\right)$ has no interior points. So we have by $[15$, Corollary 8$]$

$$
\sigma(T)=\sigma\left(T_{1}\right) \cup \sigma\left(T_{3}\right)=\sigma\left(T_{1}\right) \cup\{0\} .
$$

(ii) By [1, Lemma 1.21], it is well known that the approximate spectrum of $T_{1}$ lies in unit circle. Hence $0 \notin \sigma_{a p}\left(T_{1}\right)$. Consequently, $T_{1}$ is bounded from below.

(iii) The proof follows from [1, Theorem 2.2].

Recall that two operators $T \in \mathcal{L}(\mathcal{H})$ and $S \in \mathcal{L}(\mathcal{H})$ are similar if there exists an invertible operator $X \in \mathcal{L}(\mathcal{H})$ such that $X T=S X$ (i.e., $T=X^{-1} S X$ or $S=X T X^{-1}$ ).

Corollary 2.6. Let $T \in \mathcal{L}(\mathcal{H})$ be an n-quasi-m-isometric operator. If $T_{1}=\left.T\right|_{\overline{\mathcal{R}\left(T^{n}\right)}}$ is invertible, then $T$ is similar to a direct sum of an $m$-isometric operator and a nilpotent operator.

Proof. By Theorem 2.4 we write the matrix representation of $T$ on $\mathcal{H}=\overline{\mathcal{R}\left(T^{n}\right)} \oplus \mathcal{N}\left(T^{* n}\right)$ as follows $T=\left(\begin{array}{cc}T_{1} & T_{2} \\ 0 & T_{3}\end{array}\right)$ where $T_{1}=\left.T\right|_{\overline{\mathcal{R}\left(T^{n}\right)}}$ is an $m$-isometric operator and $T_{3}^{n}=0$. Since $T_{1}$ is invertible, we have $\sigma\left(T_{1}\right) \cap \sigma\left(T_{3}\right)=\emptyset$. Then there exists an operator $A$ such that $T_{1} A-A T_{3}=T_{2}$ by [23]. Hence

$$
T=\left(\begin{array}{cc}
T_{1} & T_{2} \\
0 & T_{3}
\end{array}\right)=\left(\begin{array}{cc}
I & A \\
0 & I
\end{array}\right)^{-1}\left(\begin{array}{cc}
T_{1} & 0 \\
0 & T_{3}
\end{array}\right)\left(\begin{array}{cc}
I & A \\
0 & I
\end{array}\right) .
$$

The desired result follows from Theorem 2.4.

Clearly, every $n$-quasi- $m$-isometric operator is an $(n+1)$-quasi- $m$-isometric operator. In [18, Theorem 2.4], S. Mechri and S. M. Patel proved that if $T$ is a quasi-2-isometry, then $T$ is quasi- $m$-isometry for all $m \geq 2$. In the following corollary, we give a generalization that every $n$-quasi- $m$-isometric operator is an $n$-quasi- $k$-isometric operator for $k \geq m$.

Corollary 2.7. Let $T \in \mathcal{L}(\mathcal{H})$. If $T$ is an n-quasi-m-isometric operator, then $T$ is an $n$-quasi-k-isometric operator for every positive integer $k \geq m$.

Proof. If $\mathcal{R}\left(T^{n}\right)$ is dense, then $T$ is an $m$-isometric operator. Hence $T$ is a $k$-isometric operator for every positive integer $k \geq m$.

If $\mathcal{R}\left(T^{n}\right)$ is not dense, by Theorem 2.4 we write the matrix representation of $\mathrm{T}$ on $\mathcal{H}=\overline{\mathcal{R}\left(T^{n}\right)} \oplus \mathcal{N}\left(T^{* n}\right)$ as follows $T=\left(\begin{array}{cc}T_{1} & T_{2} \\ 0 & T_{3}\end{array}\right)$ where $T_{1}=\left.T\right|_{\overline{\mathcal{R}\left(T^{n}\right)}}$ is an $m$-isometric operator and $T_{3}^{n}=0$. Obviously, $T_{1}$ is a $k$-isometric operator for every integer $k \geq m$. The conclusion follows from Theorem 2.4(2). 
We consider the following example of $n$-quasi- $m$-isometric operator, which is not a quasi$m$-isometry.

Example 2.8. Let $\left(e_{k}\right)_{k \in \mathbb{N}}$ be an orthonormal basis of $\mathcal{H}$. Define $T \in \mathcal{L}(\mathcal{H})$ as follows

$$
T e_{1}=2 e_{2}, T e_{2}=3 e_{3} \quad \text { and } T e_{k}=e_{k+1} \quad \text { for } k \geq 3 \text {. }
$$

Then by a straightforward calculation, one can show

$$
\left\|T^{2} e_{k}\right\|^{2}-2\left\|T^{3} e_{k}\right\|^{2}+\left\|T^{4} e_{k}\right\|^{2}=0 \quad \forall k=1,2, \cdots,
$$

and

$$
\left\|T e_{1}\right\|^{2}-2\left\|T^{2} e_{1}\right\|^{2}+\left\|T^{3} e_{1}\right\|^{2} \neq 0 .
$$

Therefore $T$ is a 2-quasi-2-isometry but it is not a quasi-2-isometry.

In the following theorem, we give a sufficient condition such that $n$-quasi- $m$-isometric operator for $n \geq 2$ to be a quasi- $m$-isometric operator.

Theorem 2.9. Let $T \in \mathcal{L}(\mathcal{H})$ be an n-quasi-m-isometry for $n \geq 2$. If $\mathcal{N}\left(T^{* p}\right)=$ $\mathcal{N}\left(T^{*(p+1)}\right)$ for some $1 \leq p \leq n-1$, then $T$ is a $p$-quasi-m-isometry.

Proof. From the assumption $\mathcal{N}\left(T^{* p}\right)=\mathcal{N}\left(T^{*(p+1)}\right)$, it follows that $\mathcal{N}\left(T^{* p}\right)=\mathcal{N}\left(T^{* n}\right)$. Since $T$ is an $n$-quasi- $m$-isometry, we have

$$
T^{* n}\left(\sum_{0 \leq k \leq m}(-1)^{m-k}\left(\begin{array}{c}
m \\
k
\end{array}\right) T^{* k} T^{k}\right) T^{n}=0,
$$

we deduce that

$$
T^{* p}\left(\sum_{0 \leq k \leq m}(-1)^{m-k}\left(\begin{array}{c}
m \\
k
\end{array}\right) T^{* k} T^{k}\right) T^{n}=0 .
$$

This means that

$$
\begin{aligned}
0 & =\left(T^{* p}\left(\sum_{0 \leq k \leq m}(-1)^{m-k}\left(\begin{array}{c}
m \\
k
\end{array}\right) T^{* k} T^{k}\right) T^{n}\right)^{*} \\
& =T^{* n}\left(\sum_{0 \leq k \leq m}(-1)^{m-k}\left(\begin{array}{c}
m \\
k
\end{array}\right)\left(T^{* k} T^{k}\right)^{*}\right) T^{p} \\
& =0 .
\end{aligned}
$$

Using again the condition $\mathcal{N}\left(T^{* p}\right)=\mathcal{N}\left(T^{*(p+1)}\right)$, we obtain

$$
T^{* p}\left(\sum_{0 \leq k \leq m}(-1)^{m-k}\left(\begin{array}{c}
m \\
k
\end{array}\right) T^{* k} T^{k}\right) T^{p}=0 .
$$

Hence $T$ is a $p$-quasi- $m$-isometric operator.

Remark 2.10. The following example shows that Theorem 2.9 is not necessarily true if $\mathcal{N}\left(T^{* p}\right) \neq \mathcal{N}\left(T^{*(p+1)}\right)$.

Example 2.11. Consider the operator $T=\left(\begin{array}{ll}0 & 1 \\ 0 & 0\end{array}\right)$ acting on the two dimensional Hilbert space $\mathbb{C}^{2}$. Then by a straightforward calculation, one can show that $T$ is a 2 quasi-isometry but it is not a quasi-isometry. However $\mathcal{N}\left(T^{*}\right) \neq \mathcal{N}\left(T^{* 2}\right)$. 
Patel [21, Theorem 2.1], proved that any power of a 2-isometry is again a 2-isometry. T. Bermúdez et al. [6, Theorem 3.1] proved that any power of $(m, p)$-isometry is an $(m, p)$-isometry. Later S. Mechri and S. M. Patel [18] gave a partial generalization to quasi-2-isometry. The following theorem shows that any power of an $n$-quasi- $m$-isometry is an $n$-quasi- $m$-isometry.

Theorem 2.12. Let $T \in \mathcal{L}(\mathcal{H})$. Suppose $T$ is an n-quasi-m-isometric operator. Then $T^{k}$ is $n$-quasi-m-isometric operator for any $k \in \mathbb{N}$.

Proof. Two different proofs of this statement will be given.

First proof. Suppose that $T$ is $n$-quasi- $m$-isometric operator. By Lemma $2.1, T$ is $m$ isometric on $\overline{\mathcal{R}\left(T^{n}\right)}$. Therefore, in view of [6, Theorem 3.1], the operator $T^{k}$ is $m$-isometry on $\overline{\mathcal{R}\left(T^{n}\right)}$. Thus

$$
<\beta_{m}\left(T^{k}\right) x \mid x>=0, \quad \forall x \in \overline{\mathcal{R}\left(T^{n}\right)} .
$$

Using the inclusion

$$
\overline{\mathcal{R}\left(\left(T^{k}\right)^{n}\right)} \subset \overline{\mathcal{R}\left(T^{n}\right)},
$$

we get

$$
<\beta_{m}\left(T^{k}\right) x \mid x>=0, \quad \forall x \in \overline{\mathcal{R}\left(\left(T^{k}\right)^{n}\right)} .
$$

Hence $T^{k}$ is $m$-isometry on $\overline{\mathcal{R}\left(\left(T^{k}\right)^{n}\right)}$. This shows, by Lemma 2.1 , that $T^{k}$ is $n$-quasi- $m$ isometric.

Second proof. If $\mathcal{R}\left(T^{n}\right)$ is dense, then $T$ is an $m$-isometric operator and hence $T^{k}$ is $m$-isometry (by [6, Theorem 3.1]). If $\mathcal{R}\left(T^{n}\right)$ is not dense, by Theorem 2.4 we write the matrix representation of $T$ on $\mathcal{H}=\overline{\mathcal{R}\left(T^{n}\right)} \oplus \mathcal{N}\left(T^{* n}\right)$ as follows $T=\left(\begin{array}{cc}T_{1} & T_{2} \\ 0 & T_{3}\end{array}\right)$ where $T_{1}=T_{\mid \overline{\mathcal{R}\left(T^{n}\right)}}$ is an $m$-isometric and $T_{3}^{n}=0$. We notice that

$$
T^{k}=\left(\begin{array}{cc}
T_{1}^{k} & \sum_{j=0}^{k-1} T_{1}^{j} T_{2} T_{3}^{k-1-j} \\
0 & T_{3}^{k}
\end{array}\right),
$$

where $T_{1}^{k}$ is $m$-isometry and $\left(T_{3}^{k}\right)^{n}=0$. Hence $T^{k}$ is an $n$-quasi- $m$-isometric operator by Theorem 2.4.

Remark 2.13. The converse of Theorem 2.12 is not true in general as shown in the following example.

Example 2.14. It is not difficult to prove that the operator $T:=\left(\begin{array}{cc}-1 & -1 \\ 3 & 2\end{array}\right)$ defined in $\mathbb{C}^{2}$ with the Euclidean norm satisfies $T^{3}$ is a quasi-3-isometry but $T$ is not a quasi-3isometry.

The following theorem generalizes [6, Theorem 3.6].

Theorem 2.15. Let $T \in \mathcal{L}(\mathcal{H})$ and $r, s, m, n_{1}, n_{2}, l$ be positive integers. If $T^{r}$ is an $n_{1}$-quasi-m-isometry and $T^{s}$ is an $n_{2}$-quasi-l-isometry, then $T^{q}$ is an n-quasi-p-isometry, where $q$ is the greatest common divisor of $r$ and $s, n=\frac{1}{q} \min \left(n_{1} r, n_{2} s\right)$ and $p=\min (m, l)$.

Proof. Consider the matrix representation of $T$ with respect to the decomposition $\mathcal{H}=\overline{\mathcal{R}\left(T^{n}\right)} \oplus \mathcal{N}\left(T^{* n}\right)$ as follows $T=\left(\begin{array}{cc}T_{1} & T_{2} \\ 0 & T_{3}\end{array}\right)$ where $T_{1}=\left.T\right|_{\overline{\mathcal{R}\left(T^{n}\right)}}$ is $m$-isometric and $T_{3}^{n}=0$. 
We have $T^{r}=\left(\begin{array}{cc}T_{1}^{r} & \sum_{j=0}^{r-1} T_{1}^{j} T_{2} T_{3}^{r-1-j} \\ 0 & T_{3}^{r}\end{array}\right)$. Since $T^{r}$ is an $n$-quasi- $m$-isometry, we need to prove that $T_{1}^{r}$ is an $m$-isometry and $\left(T_{3}^{r}\right)^{n}=0$.

In fact, let $P=P_{\overline{\mathcal{R}\left(T^{n}\right)}}$ be the projection on $\overline{\mathcal{R}\left(T^{n}\right)}$. Then

$$
\left(\begin{array}{cc}
T_{1}^{r} & 0 \\
0 & 0
\end{array}\right)=T^{r} P=P T^{r} P
$$

Since $T^{r}$ is an $n$-quasi- $m$-isometry, we have

$$
P\left(\sum_{0 \leq k \leq m}(-1)^{m-k}\left(\begin{array}{c}
m \\
k
\end{array}\right)\left(T^{r}\right)^{* k}\left(T^{r}\right)^{k}\right) P=0,
$$

that is,

$$
\sum_{0 \leq k \leq m}(-1)^{m-k}\left(\begin{array}{c}
m \\
k
\end{array}\right)\left(T_{1}^{r}\right)^{* k}\left(T_{1}^{r}\right)^{k}=0 .
$$

Hence, $T_{1}^{r}$ is an $m$-isometry.

On the other hand, let $x=x_{1}+x_{2} \in \mathcal{H}=\overline{\mathcal{R}\left(T^{n}\right)} \oplus \mathcal{N}\left(T^{* n}\right)$. A simple computation shows that

$$
\left\langle\left(T_{3}^{r}\right)^{n} x_{2}, x_{2}\right\rangle=\left\langle\left(T^{r}\right)^{n}(I-P) x,(I-P) x\right\rangle=\left\langle(I-P) x,\left(T^{r}\right)^{* n}(I-P) x\right\rangle=0 .
$$

So, $\left(T_{3}^{r}\right)^{n}=0$.

Analogously, as $T^{s}=\left(\begin{array}{cc}T_{1}^{s} & \sum_{j=0}^{s-1} T_{1}^{j} T_{2} T_{3}^{s-1-j} \\ 0 & T_{3}^{s}\end{array}\right)$ is an $n$-quasi-l-isometry by similar arguments, we can conclude that $T_{1}^{s}$ is an $l$-isometry and $\left(T_{3}^{s}\right)^{n}=0$.

Now, we have obtained that $T_{1}^{r}$ is an $m$-isometry and $T_{1}^{s}$ is an $l$-isometry. By $[6$, Theorem 3.6], it follows that $T_{1}^{q}$ is a $p=\min (m, l)$-isometry. Moreover we have

$$
\left(T_{3}^{r}\right)^{n_{1}}=0=\left(T_{3}^{q}\right)^{\frac{r}{q} n_{1}} \text { and }\left(T_{3}^{s}\right)^{n_{2}}=0=\left(T_{3}^{q}\right)^{\frac{s}{q} n_{2}} .
$$

Since $n=\frac{1}{q} \min \left(n_{1} r, n_{2} s\right)$, we obtain $\left(T_{3}^{q}\right)^{n}=0$.

Consequently, $T^{q}=\left(\begin{array}{cc}T_{1}^{q} & \sum_{j=0}^{q-1} T_{1}^{j} T_{2} T_{3}^{q-1-j} \\ 0 & T_{3}^{q}\end{array}\right)$ is an $n$-quasi- $p$-isometry by Theorem 2.4. The proof is completed.

The following corollary shows that if two suitable different powers of $T$ are $n$-quasi-misometries, then $T$ is a $n$-quasi- $m$-isometry.

Corollary 2.16. Let $T \in \mathcal{L}(\mathcal{H})$ and $r, s, m, n, l$ be positive integers. The following properties hold.

(1) If $T$ is an n-quasi-m-isometry such that $T^{s}$ is an n-quasi-isometry, then $T$ is an n-quasi-isometry.

(2) If $T^{r}$ and $T^{r+1}$ are $n$-quasi-m-isometries, then $T$ is a nr-quasi-m-isometry.

(3) If $T^{r}$ is an $n$-quasi-m-isometry and $T^{r+1}$ is an $n$-quasi-l-isometry with $m<l$, then $T$ is an $n r$-quasi-m-isometry. 
Proof. The proof is an immediate consequence of Theorem 2.15 .

Recall that an operator $T \in \mathcal{L}(\mathcal{H})$ is said to be power bounded, if $\sup _{k}\left\|T^{k}\right\|<\infty$ or equivalently, there exists $C>0$ such that for every $k$ and every $\xi \in \mathcal{H}$, one has

$$
\left\|T^{k} \xi\right\| \leq C\|\xi\| \text {. }
$$

In [8, Theorem 2], it was proved that every power bounded $m$-isometry operator is an isometry. The following theorem extends this result to $n$-quasi- $m$-isometry.

Theorem 2.17. If $T \in \mathcal{L}(\mathcal{H})$ is an n-quasi-m-isometric operator which is power bounded, then $T$ is an n-quasi-isometry.

Proof. We consider the following two cases:

Case 1: If $\overline{\mathcal{R}\left(T^{n}\right)}$ is dense, then $T$ is an $m$-isometric operator which is power bounded, thus $T$ is an isometry by [8, Theorem 2]. It follows that $T$ is an $n$-quasi-isometry.

Case 2: If $\overline{\mathcal{R}\left(T^{n}\right)}$ is not dense, by Theorem 2.4 we write the matrix representation of $T$ on $\mathcal{H}=\overline{\mathcal{R}\left(T^{n}\right)} \oplus \mathcal{N}\left(T^{* n}\right)$ as follows $T=\left(\begin{array}{cc}T_{1} & T_{2} \\ 0 & T_{3}\end{array}\right)$ where $T_{1}=\left.T\right|_{\overline{\mathcal{R}\left(T^{n}\right)}}$ is an $m$-isometric operator and $T_{3}^{n}=0$. By taking into account that $T$ is power bounded, it is easily checked that $T_{1}$ is power bounded, from which we deduce that $T_{1}$ is an isometry. The result follows by applying Theorem 2.4(2).

Recall that for two operators $T, S$ in $\mathcal{L}(\mathcal{H})$, the commutator $[T, S]$ is defined to be

$$
[T, S]=T S-S T \text {. }
$$

A pair of operators $(T, S) \in \mathcal{L}(\mathcal{H})^{2}$ is said to be a doubly commuting pair if $(T, S)$ satisfies $T S=S T$ and $T^{*} S=S T^{*}$ or equivalently $[T, S]=\left[T, S^{*}\right]=0$.

In [16, Theorem 2.2], it was proved that if $T$ and $S$ are commuting bounded linear operators on a Banach space such that $T$ is a 2-isometry and $S$ is an $m$-isometry, then $S T$ is an $(m+1)$-isometry. This result was improved in [3, Theorem 3.3] as follows: if $T S=S T, T$ is an $(m, p)$-isometry and $S$ is an $(l, p)$-isometry, then $S T$ is an $(m+l-1, p)$ isometry. It is natural to ask whether the product of two $n$-quasi- $m$-isometries is also $n$-quasi- $m$-isometry. The following theorem gives an affirmative answer under suitable conditions.

Theorem 2.18. Let $S$ and $T$ in $\mathcal{L}(\mathcal{H})$ be doubly commuting operators and let $m, l, n_{1}, n_{2}$ be positive integers. If $T$ is an $n_{1}$-quasi-m-isometry and $S$ is an $n_{2}$-quasi-l-isometry, then $T S$ is a $n_{0}=\max \left\{n_{1}, n_{2}\right\}$-quasi- $(m+l-1)$-isometry.

Proof. Since $T$ and $S$ are doubly commuting, it follows that $\left[T^{*}, S^{*}\right]=[T, S]=\left[T, S^{*}\right]=$ 0 . By taking into account [11, Lemma 12] we obtain that

$$
\begin{aligned}
& \beta_{m+l-1, n_{0}}(T S) \\
& =(T S)^{*\left(n_{0}\right)} \beta_{m+l-1}(T S)(T S)^{n_{0}} \\
& =\left(T^{*}\right)^{n_{0}}\left(S^{*}\right)^{n_{0}} \beta_{m+l-1}(T S)(T)^{n_{0}}(S)^{n_{0}} \\
& =\left(T^{*}\right)^{n_{0}}\left(S^{*}\right)^{n_{0}}\left(\sum_{0 \leq j \leq m+l-1}\left(\begin{array}{c}
m+l-1 \\
j
\end{array}\right) T^{* j} \beta_{m+l-1-j}(T) T^{j} \beta_{j}(S)\right)(T)^{n_{0}}(S)^{n_{0}} \\
& =(\sum_{0 \leq j \leq m+l-1}\left(\begin{array}{c}
m+l-1 \\
j
\end{array}\right) T^{* j} \underbrace{\left(T^{*}\right)^{n_{0}} \beta_{m+l-1-j}(T) T^{n_{0}}} T^{j} \underbrace{\left(S^{*}\right)^{n_{0}} \beta_{j}(S) S^{n_{0}}}) .
\end{aligned}
$$


Since $S$ is an $n_{2}$-quasi- $l$-isometry, it follows from Corollary 2.7 that $\left(S^{*}\right)^{n_{0}} \beta_{j}(S) S^{n_{0}}=0$ for $j \geq l$. On the other hand, if $j \leq l-1$, then $m+l-1-j \geq m+l-1-l+1=m$, and so $\left(T^{*}\right)^{n_{0}} \beta_{k+m-1-j}(T) T^{n_{0}}=0$ by the fact that $T$ is an $n_{1}$-quasi- $m$-isometry. This completes the proof.

The following example shows that Theorem 2.18 is not necessarily true if $S, T$ are not doubly commuting.

Example 2.19. We consider the operators $T=\left(\begin{array}{ll}1 & 1 \\ 0 & 1\end{array}\right)$ and $S=\left(\begin{array}{cc}2 & 1 \\ -1 & 0\end{array}\right)$ on the two dimensional Hilbert space $\mathbb{C}^{2}$. Note that $S T \neq T S$. Moreover, by a direct computation, we show that $T$ is a quasi-3-isometry and $S$ is a 2-quasi-3-isometry. However neither $T S$ nor $S T$ is a 2 -quasi-5-isometry.

Corollary 2.20. Let $T, S \in \mathcal{L}(\mathcal{H})$ be doubly commuting operators such that $T$ is an $n_{1}$-quasi-m-isometry and $S$ is an $n_{2}$-quasi-l-isometry, then $T^{p} S^{q}$ is a $\max \left\{n_{1}, n_{2}\right\}$-quasi$(m+l-1)$-isometry for all positive integers $p$ and $q$.

Proof. Since $T$ and $S$ are doubly commuting, then $T^{p}$ and $S^{q}$ are doubly commuting. By Theorem 2.12 we know that $T^{p}$ is an $n_{1}$-quasi- $m$-isometry and $S^{q}$ is an $n_{2}$-quasi-l-isometry. Now by applying Theorem 2.18 , we get that $T^{p} S^{q}$ is a $\max \left\{n_{1}, n_{2}\right\}$-quasi- $(m+l-1)$ isometry. This completes the proof.

Let $\mathcal{H} \bar{\otimes} \mathcal{H}$ denote the completion, endowed with a reasonable uniform cross-norm, of the algebraic tensor product $\mathcal{H} \otimes \mathcal{H}$ of $\mathcal{H}$ and $\mathcal{H}$. For $T \in \mathcal{L}(\mathcal{H})$ and $S \in \mathcal{L}(\mathcal{H}), T \otimes S \in$ $\mathcal{L}(\mathcal{H} \bar{\otimes} \mathcal{H})$ denote the tensor product operator defined by $T$ and $S$.

In the following proposition we prove that the tensor product of an $n_{1}$-quasi- $m$-isometric operator with an $n_{2}$-quasi- $l$-isometric operator is a $\max \left\{n_{1}, n_{2}\right\}$-quasi- $(m+l-1)$-isometric operator. This proposition generalizes [9, Theorem 2.10].

Proposition 2.21. If $T \in \mathcal{L}(\mathcal{H})$ is an $n_{1}$-quasi-m-isometry and $S \in \mathcal{L}(\mathcal{H})$ is an $n_{2}$-quasi$l$-isometry, then $T \otimes S$ is a $\max \left\{n_{1}, n_{2}\right\}$-quasi- $(m+l-1)$-isometric operator.

Proof. Observe that an operator $T \in \mathcal{L}(\mathcal{H})$ is $n$-quasi $m$-isometric if and only if $T \otimes I$ and $I \otimes T$ are $n$-quasi- $m$-isometry. In view of the fact that

$$
T \otimes S=(T \otimes I)(I \otimes S)=(I \otimes S)(T \otimes I)
$$

it follows that

$$
[T \otimes I, I \otimes S]=\left[T \otimes I,(I \otimes S)^{*}\right]=0 .
$$

Now $T \otimes I$ is an $n_{1}$-quasi- $m$-isometry and $I \otimes S$ is an $n_{2}$-quasi- $m$-isometry such that $T \otimes I$ and $I \otimes S$ are doubly commuting operators. By applying Theorem 2.18 we obtain that $(T \otimes I)(I \otimes S)$ is a $\max \left\{n_{1}, n_{2}\right\}$-quasi- $(m+l-1)$-isometric operator. Hence $T \otimes S$ is a $\max \left\{n_{1}, n_{2}\right\}$-quasi- $(m+l-1)$-isometric as required.

The following corollary is an immediate consequence of Theorem 2.12 and Proposition 2.21. We omitted its proof.

Corollary 2.22. If $T \in \mathcal{L}(\mathcal{H})$ is an $n_{1}$-quasi-m-isometry and $S \in \mathcal{L}(\mathcal{H})$ is an $n_{2}$-quasil-isometry, then $T^{p} \otimes S^{q}$ is a $\max \left\{n_{1}, n_{2}\right\}$-quasi- $(m+l-1)$-isometry.

It was proved in [4, Thoerem 2.2] that if $T \in \mathcal{L}(\mathcal{H})$ is an isometry and $Q \in \mathcal{L}(\mathcal{H})$ is a nilpotent operator of order $p$ such that $T Q=Q T$, then $T+Q$ is a strict $(2 p-1)$-isometry. Later T. Bermúdez et al. [2] gave a partial generalization to $m$-isometry, that is if $T$ is an 
$m$-isometry with $m>1, Q$ is a nilpotent operator with order $p$, and $T Q=Q T$, then $T+Q$ is an $(m+2 p-2)$-isometry. Recently, C. Gu. and M. Stankus [14] gave a generalization to $m$-isometry, that is, if $T$ is an $m$-isometry with $m>1, Q$ is a nilpotent operator with order $p$, and $T Q=Q T$, then $T+Q$ is a strict $(m+2 p-2)$-isometry. The following theorem states the corresponding partial generalization to the sum of an $n$-quasi- $m$-isometry and a nilpotent operator.

Theorem 2.23. Let $T, Q \in \mathcal{L}(\mathcal{H})$ such that $T$ commutes with $Q$. If $T$ is an $n$-quasi-misometry and $Q$ is a nilpotent operator of order $p$, then $T+Q$ is a $(n+p)$-quasi- $(m+2 p-2)$ isometry.

Proof. We need to show that $\beta_{m+2 p-2, \alpha}(T+Q)=0$. Set $q=m+2 p-2$ and $\alpha=n+p$, by $[14$, Lemma 1] we have

$$
\beta_{q}(T+Q)=\sum_{0 \leq k \leq q} \sum_{0 \leq j \leq q-k}\left(\begin{array}{c}
q \\
k
\end{array}\right)\left(\begin{array}{c}
q-k \\
j
\end{array}\right)\left(T^{*}+Q^{*}\right)^{k} Q^{* j} \beta_{q-k-j}(T) T^{j} Q^{k} .
$$

In fact, note that

$$
\begin{aligned}
& \beta_{m+2 p-2, \alpha}(T+Q) \\
&=(T+Q)^{* \alpha} \beta_{m+2 p-2}(T+Q)(T+Q)^{\alpha} \\
&=\left(\sum_{0 \leq r \leq \alpha}\left(\begin{array}{l}
\alpha \\
r
\end{array}\right) T^{*(\alpha-r)} Q^{* r}\right)\left(\sum_{0 \leq k \leq q} \sum_{0 \leq j \leq q-k}\left(\begin{array}{c}
q \\
k
\end{array}\right)\left(\begin{array}{c}
q-k \\
j
\end{array}\right)\left(T^{*}+Q^{*}\right)^{k} Q^{* j} \beta_{q-k-j}(T) T^{j} Q^{k}\right) \\
& \times\left(\sum_{0 \leq r \leq \alpha}\left(\begin{array}{l}
\alpha \\
r
\end{array}\right) T^{\alpha-r} Q^{r}\right) .
\end{aligned}
$$

Now observe that if $k \geq p$ or $j \geq p$ then $Q^{k}=0$ or $Q^{* j}=0$ and hence

$$
\left(T^{*}+Q^{*}\right)^{k} Q^{* j} \beta_{q-k-j}(T) T^{j} Q^{k}=0 .
$$

However, if $k<p$ and $j<p$, we obtain

$$
q-k-j=m+2 p-2-k-j \geq m+2 p-2-(p-1)-(p-1)=m
$$

and using the fact that $T$ is an $n$-quasi- $m$-isometry, we get

$$
T^{*(n+p-r)} \beta_{q-k-j}(T) T^{n+p-r}=0 \text { for } r=0, \cdots, p
$$

and

$$
T^{*(n+p-r)} Q^{* r} \beta_{q-k-j}(T) T^{n+p-r} Q^{r}=0 \text { for } r=p+1, \cdots, n+p .
$$

Combining the above arguments we obtain $\beta_{m+2 p-2, n+p}(T+Q)=0$.

Remark 2.24. A simple example shows that the commuting condition of $T$ and $Q$ can not be removed from the above theorem.

Example 2.25. Let $T=\left(\begin{array}{cc}-5 & 0 \\ 0 & -1\end{array}\right)$ and $Q=\left(\begin{array}{ll}0 & 1 \\ 0 & 0\end{array}\right)$. Then $T$ is a quasi-3-isometry and $Q^{2}=0$. Set $S=T+Q$, by direct calculation we show that $S$ is not 5-quasi-5-isometry.

Corollary 2.26. Let $T \in \mathcal{L}(\mathcal{H})$ be an n-quasi-m-isometry and $Q \in \mathcal{L}(\mathcal{H})$ be a nilpotent operator of order $p$. Then $T \otimes I+I \otimes Q$ is a $(n+p)$-quasi- $(m+2 p-2)$-isometry.

Proof. We note that $T \otimes I \in \mathcal{L}(\mathcal{H} \bar{\otimes} \mathcal{H})$ is an $n$-quasi- $m$-isometry and $I \otimes Q \in \mathcal{L}(\mathcal{H} \bar{\otimes} \mathcal{H})$ is nilpotent of order $p$. Moreover $(T \otimes I)(I \otimes Q)=(I \otimes Q)(T \otimes I)$. 
The following theorem shows that the class of $n$-quasi- $m$-isometry is a closed subset of $\mathcal{L}(\mathcal{H})$ equipped with the uniform operator (norm) topology.

Theorem 2.27. Let $T \in \mathcal{L}(\mathcal{H})$. If $\left(T_{k}\right)_{k}$ is a sequence of $n$-quasi-m-isometry such that $\lim _{k \rightarrow \infty}\left\|T_{k}-T\right\|=0$, then $T$ is also $n$-quasi-m-isometry.

Proof. Suppose that $\left(T_{k}\right)_{k}$ is a sequence of $n$-quasi- $m$-isometric operators such that

$$
\lim _{k \rightarrow \infty}\left\|T_{k}-T\right\|=0 .
$$

Since for every positive integer $k, T_{k}$ is an $n$-quasi- $m$-isometry, we have $\beta_{m, n}\left(T_{k}\right)=0$. It follows that

$$
\begin{aligned}
& \left\|\beta_{m, n}(T)\right\|=\left\|\beta_{m, n}\left(T_{k}\right)-\beta_{m, n}(T)\right\| \\
= & \left\|T_{k}^{* n}\left(\sum_{0 \leq j \leq m}(-1)^{m-j}\left(\begin{array}{c}
m \\
j
\end{array}\right) T_{k}^{* j} T_{k}^{j}\right) T_{k}^{n}-T^{* n}\left(\sum_{0 \leq j \leq m}(-1)^{m-j}\left(\begin{array}{c}
m \\
j
\end{array}\right) T^{* j} T^{j}\right) T^{n}\right\| \\
\leq & \left\|T_{k}^{* n}\left(\sum_{0 \leq j \leq m}(-1)^{m-j}\left(\begin{array}{c}
m \\
j
\end{array}\right) T_{k}^{* j} T_{k}^{j}\right) T_{k}^{n}-T_{k}^{* n}\left(\sum_{0 \leq j \leq m}(-1)^{m-j}\left(\begin{array}{c}
m \\
j
\end{array}\right) T_{k}^{* j} T^{j}\right) T^{n}\right\| \\
& +\left\|T_{k}^{* n}\left(\sum_{0 \leq j \leq m}(-1)^{m-j}\left(\begin{array}{c}
m \\
j
\end{array}\right) T_{k}^{* j} T^{j}\right) T^{n}-T^{* n}\left(\sum_{0 \leq j \leq m}(-1)^{m-j}\left(\begin{array}{c}
m \\
j
\end{array}\right) T^{* j} T^{j}\right) T^{n}\right\| \\
\leq & \left\|\sum_{0 \leq j \leq m}(-1)^{m-j}\left(\begin{array}{c}
m \\
j
\end{array}\right) T_{k}^{* n+j}\left(T_{k}^{j} T_{k}^{n}-T^{j} T^{n}\right)\right\| \\
& +\left\|\sum_{0 \leq j \leq m}(-1)^{m-j}\left(\begin{array}{c}
m \\
j
\end{array}\right)\left(T_{k}^{* n+j}-T^{* n+j}\right) T^{j} T^{n}\right\| \\
\leq & \sum_{0 \leq j \leq m}\left(\begin{array}{c}
m \\
j
\end{array}\right)\left\|T_{k}^{*}\right\|^{n+j}\left\|T_{k}^{j} T_{k}^{n}-T^{j} T^{n}\right\|+\sum_{0 \leq j \leq m}\left(\begin{array}{c}
m \\
j
\end{array}\right)\left\|T_{k}^{* n+j}-T^{n+j}\right\|\|T\|^{j+n} .
\end{aligned}
$$

Since the product of operators is sequentially continuous in the strong topology, one concludes that $T_{k}^{j} T_{k}^{n}, T_{k}^{n+j}$ converges strongly to $T^{j} T^{n}$ and $T^{n+j}$ respectively for $j=$ $0,1, \ldots, m$. Hence the limiting case of $(2.5)$ shows that $T$ belongs to the class of $n$-quasi$m$-isometric operators.

\section{3. $n$-quasi strict- $m$-isometries}

In this section we introduce and study some properties of the class of $n$-quasi strict$m$-isometric operators.

Recall that an operator $T \in \mathcal{L}(\mathcal{H})$ is said to be a strict $m$-isometry if $T$ is an $m$-isometry but it is not an $(m-1)$-isometry.

Definition 3.1. We say that $T \in \mathcal{L}(\mathcal{H})$ is a $n$-quasi strict $m$-isometry if $T$ is an $n$-quasi$m$-isometry, but $T$ is not an $n$-quasi- $(m-1)$-isometry.

Example $3.2([18])$. Let $\left(e_{k}\right)_{k \geq 1}$ be an orthonormal basis of a Hilbert space $\mathcal{H}$. Consider an operator $T \in \mathcal{L}(\mathcal{H})$ defined by:

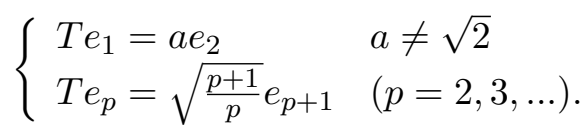

A direct calculation shows that $T$ is a quasi-2-isometric operator, but not a quasi-isometric operator. Therefore $T$ is a quasi strict-2-isometry. 
Example 3.3. Consider the operator $T \in \mathcal{L}\left(\mathbb{C}^{2}\right)$ given by $T=\left(\begin{array}{ll}1 & 1 \\ 0 & 1\end{array}\right)$ which is quasi3 -isometric operator but is not quasi-2-isometric operator. Hence $T$ is a quasi strict-3isometry.

Remark 3.4. It is proved in Corollary 2.7 that an $n$-quasi- $m$-isometric operator is $n$ quasi- $k$-isometric operator for all integers $k \geq m$. Hence if an $T \in \mathcal{L}(\mathcal{H})$ is a strict $n$ quasi- $m$-isometry, then it is not a $n$-quasi- $k$-isometry for all integers $1 \leq k<m$.

Recall that the multinomial coefficient is given by $\left(\begin{array}{c}k \\ p_{1}, \cdots, p_{k}\end{array}\right)=\frac{k !}{p_{1} ! p_{2} ! \cdots p_{k} !}$ where $k$ and $p_{1}, \cdots, p_{k}$ are nonnegative integers subject to $k=p_{1}+p_{2}+\cdots+p_{k}$.

We will use the following formula for commuting variables $z=\left(z_{1}, \ldots, z_{q}\right)$ :

$$
\left(z_{1}+\cdots+z_{q}\right)^{k}=\sum_{p_{1}+p_{2}+\cdots+p_{q}=k}\left(\begin{array}{c}
k \\
p_{1}, p_{2}, \cdots, p_{q}
\end{array}\right) z_{1}^{p_{1}} z_{2}^{p_{2}} \cdots z_{q}^{p_{q}} .
$$

In particular, if $z_{1}=\cdots=z_{q}=1$, we have

$$
\sum_{p_{1}+p_{2}+\cdots+p_{q}=k}\left(\begin{array}{c}
k \\
p_{1}, p_{2}, \cdots, p_{q}
\end{array}\right)=q^{k} \text {. }
$$

Proposition 3.5. Let $T \in \mathcal{L}(\mathcal{H})$. Then the following statements hold.

(1) If $m$ is a positive integer and $x \in \mathcal{H}$, then

$$
\Delta_{m, n}(T, x)=\Delta_{m-1, n}(T, T x)-\Delta_{m-1, n}(T, x) .
$$

In particular, if $T$ is an n-quasi-m-isometry, then for every positive integer $k$ one has

$$
\Delta_{m-1, n}\left(T, T^{k} x\right)=\Delta_{m-1, n}(T, x) .
$$

(2) If $k$ is a positive integer and $x \in \mathcal{H}$, then

$$
\Delta_{m, n}\left(T^{k}, x\right)=\sum_{p_{1}+\cdots+p_{k}=m}\left(\begin{array}{c}
m \\
p_{1}, \cdots, p_{k}
\end{array}\right) \Delta_{m, n}\left(T, T^{\left(0 . p_{1}+1 \cdot p_{2}+\cdots+(k-1) p_{k}\right)+(k-1) n} x\right) .
$$

Proof. For $m \geq 1$ and $x \in \mathcal{H}$ we have

$$
\begin{aligned}
\Delta_{m, n}(T, x)=\sum_{0 \leq k \leq m}(-1)^{m-k}\left(\begin{array}{c}
m \\
k
\end{array}\right)\left\|T^{k+n} x\right\|^{2} \\
=(-1)^{m}\left\|T^{n} x\right\|^{2}+\sum_{1 \leq k \leq m}(-1)^{m-1-k}\left(\begin{array}{c}
m \\
k
\end{array}\right)\left\|T^{k+n} x\right\|^{2}+\left\|T^{m+n} x\right\|^{2} \\
=(-1)^{m}\left\|T^{n} x\right\|^{2}+\sum_{1 \leq k \leq m}(-1)^{m-1-k}\left(\left(\begin{array}{c}
m-1 \\
k
\end{array}\right)+\left(\begin{array}{c}
m-1 \\
k-1
\end{array}\right)\right)\left\|T^{k+n} x\right\|^{2}+\left\|T^{m+n} x\right\|^{2} \\
=\sum_{0 \leq k \leq m-1}(-1)^{m-1-k}\left(\begin{array}{c}
m-1 \\
k
\end{array}\right)\left\|T^{k+n} T x\right\|^{2}-\sum_{0 \leq k \leq m-1}(-1)^{m-1-k}\left(\begin{array}{c}
m-1 \\
k
\end{array}\right)\left\|T^{k+n} x\right\|^{2} \\
=\Delta_{m-1, n}(T, T x)-\Delta_{m-1, n}(T, x) .
\end{aligned}
$$

Hence (3.1) is proved.

If $T$ is an $n$-quasi- $m$-isometry, then $\Delta_{m, n}(T, x)=0$ and so that

$$
\Delta_{m-1, n}(T, T x)=\Delta_{m-1, n}(T, x) .
$$


Hence,

$$
\Delta_{m-1, n}\left(T, T^{k} x\right)=\Delta_{m-1, n}\left(T, T^{k-1} x\right)=\cdots=\Delta_{m-1, n}(T, x) .
$$

(2) From (2.3) we have

$$
\Delta_{m, n}\left(T^{k}, x\right)=\sum_{0 \leq j \leq m}(-1)^{m-j}\left(\begin{array}{c}
m \\
j
\end{array}\right)\left\|\left(T^{k}\right)^{j+n} x\right\|^{2}
$$

and

$$
\begin{aligned}
& \sum_{p_{1}+\cdots+p_{k}=m}\left(\begin{array}{c}
m \\
p_{1}, \cdots, p_{k}
\end{array}\right) \Delta_{m, n}\left(T, T^{\left(0 \cdot p_{1}+1 \cdot p_{2}+\cdots+(k-1) p_{k}\right)+(k-1) n} x\right)= \\
& \sum_{p_{1}+\cdots+p_{k}=m}\left(\begin{array}{c}
m \\
p_{1}, \cdots, p_{k}
\end{array}\right) \sum_{0 \leq j \leq m}(-1)^{m-j}\left(\begin{array}{c}
m \\
j
\end{array}\right)\left\|T^{j+k n} T^{\left(0 \cdot p_{1}+1 \cdot p_{2}+\cdot+(k-1) p_{k}\right)} x\right\|^{2} \cdot(3.5)
\end{aligned}
$$

Thus, for proving (3.3) it suffices to show that the coefficients of the various powers of $\left\|T^{j} x\right\|^{2}$ for $n k \leq j \leq k m+k n$ in (3.4) and (3.5) are the same. To do so, we merely need to check the following combinatorial identity:

$$
\begin{aligned}
& \sum_{0 \leq j \leq m}(-1)^{m-j}\left(\begin{array}{c}
m \\
j
\end{array}\right)\left(z^{k}\right)^{j+n} \\
& =\sum_{p_{1}+\cdots+p_{k}=m}\left(\begin{array}{c}
m \\
p_{1}, \cdots, p_{k}
\end{array}\right)\left(\sum_{0 \leq j \leq m}(-1)^{m-j}\left(\begin{array}{c}
m \\
j
\end{array}\right) z^{j+k n} z^{\left(0 . p_{1}+1 \cdot p_{2}+\cdots+(k-1) p_{k}\right)}\right) .
\end{aligned}
$$

In fact, observe that

$$
\sum_{0 \leq j \leq m}(-1)^{m-j}\left(\begin{array}{c}
m \\
j
\end{array}\right)\left(z^{k}\right)^{j+n}=\left(z^{k}-1\right)^{m} z^{k n},
$$

and

$$
\begin{aligned}
& \sum_{p_{1}+\cdots+p_{k}=m}\left(\begin{array}{c}
m \\
p_{1}, \cdots, p_{k}
\end{array}\right)\left(\sum_{0 \leq j \leq m}(-1)^{m-j}\left(\begin{array}{c}
m \\
j
\end{array}\right) z^{j+k n} z^{\left(0 . p_{1}+1 \cdot p_{2}+\cdots+(k-1) p_{k}\right)}\right) \\
= & z^{k n} \sum_{p_{1}+\cdots+p_{k}=m}\left(\begin{array}{c}
m \\
p_{1}, \cdots, p_{k}
\end{array}\right)\left(\sum_{0 \leq j \leq m}(-1)^{m-j}\left(\begin{array}{c}
m \\
j
\end{array}\right) z^{j}\right) z^{\left(0 . p_{1}+1 \cdot p_{2}+\cdots+(k-1) p_{k}\right)} .
\end{aligned}
$$

By applying the multinomial formula in reverse order, we have

$$
\begin{aligned}
& \sum_{p_{1}+\cdots+p_{k}=m}\left(\begin{array}{c}
m \\
p_{1}, \cdots, p_{k}
\end{array}\right)\left(\sum_{0 \leq j \leq m}(-1)^{m-j}\left(\begin{array}{c}
m \\
j
\end{array}\right) z^{j}\right) z^{\left(0 . p_{1}+1 \cdot p_{2}+\cdots+(k-1) p_{k}\right)} \\
= & (z-1)^{m} \sum_{p_{1}+\cdots+p_{k}=m}\left(\begin{array}{c}
m \\
p_{1}, \cdots, p_{k}
\end{array}\right) z^{\left(0 \cdot p_{1}+1 \cdot p_{2}+\cdots+(k-1) p_{k}\right)} \\
= & (z-1)^{m}\left(z^{k-1}+z^{k-2}+\cdots+z+1\right)^{m}=\left(z^{k}-1\right)^{m} .
\end{aligned}
$$

Theorem 3.6. If $T \in \mathcal{L}(\mathcal{H})$ is a n-quasi strict $m$-isometry, then for any positive integer $k, T^{k}$ is a n-quasi strict m-isometry. Furthermore

$$
\Delta_{m-1, n}\left(T^{k}, x\right)=k^{m-1} \Delta_{m-1, n}(T, x) .
$$


Proof. Since $T$ is an $n$-quasi strict $m$-isometry, by Theorem $2.12, T^{k}$ is an $n$-quasi- $m$ isometry. Furthermore, by (3.2) and (3.3) we get

$$
\begin{aligned}
\Delta_{m-1, n}\left(T^{k}, x\right) & =\sum_{p_{1}+\cdots+p_{k}=m-1}\left(\begin{array}{c}
m-1 \\
p_{1}, \cdots, p_{k}
\end{array}\right) \Delta_{m-1, n}\left(T, T^{\left(0 . p_{1}+1 . p_{2}+\cdots+(k-1) p_{k}\right)+(k-1) n} x\right) \\
& =\sum_{p_{1}+\cdots+p_{k}=m-1}\left(\begin{array}{c}
m-1 \\
p_{1}, \cdots, p_{k}
\end{array}\right) \Delta_{m-1, n}(T, x) \\
& =k^{m-1} \Delta_{m-1, n}(T, x) .
\end{aligned}
$$

Consequently, $T^{k}$ is a $n$-quasi strict- $m$-isometry.

Remark 3.7. The converse of the above theorem is not true in general. In fact, by Theorem 2.15 if $T^{r}$ and $T^{s}$ are $n$-quasi- $m$-isometries for two coprime positive integers $r$ and $s$, then $T$ is an $n$-quasi- $m$-isometry.

Recall that a sequence $\left(a_{j}\right)_{j \geq 0}$ in a group $G$ is an arithmetic progression of order $h$ if

$$
\sum_{0 \leq k \leq h+1}(-1)^{h+1-k} a_{k+j}=0
$$

for any $j \geq 0$. An arithmetic progression of order $h$ is of strict order $h$ if $h=0$ or if $h \geq 1$ and it is not of order $h-1$. We refer the interested reader to [5] for complete details.

Lemma 3.8 ([5, Theorem 4.1]). Let $a=\left(a_{j}\right)_{j \geq 0}$ be a numerical sequence. Suppose that $\left(a_{c j}\right)_{j \geq 0}$ is an arithmetic progression of strict order $h$ and $\left(a_{d j}\right)_{j \geq 0}$ is an arithmetic progressions of strict order $k \geq 0$, for $c, d \geq 1$ and $h, k \geq 0$. Then $\left(a_{e j}\right)_{j \geq 0}$ is an arithmetic progression of strict order $l$, where $e$ is the greatest common divisor of $c$ and $d$, and $l$ the minimum of $h$ and $k$.

Theorem 3.9. Let $T \in \mathcal{L}(\mathcal{H})$ and $r, s, n, m, l$ be positive integers. If $T^{r}$ is a n-quasi strict $m$-isometry and $T^{s}$ is an n-quasi strict l-isometry, then $T^{q}$ is an n-quasi strict p-isometry, where $q$ is the greatest common divisor of $r$ and $s$, and $p$ is the minimum of $m$ and $l$.

Proof. Without loss of generality, we will suppose that $r \geq s$. Fix $x \in \mathcal{H}$ and set $a_{j}:=$ $\left\|T^{j+n r} x\right\|^{2}$ for $j \geq 0$. Since $T^{r}$ is a $n$-quasi strict $m$-isometry, it follows that $\left(a_{r j}\right)_{j \geq 0}$ is an arithmetic progression of strict order $m-1$ and satisfies the recursive equation

$$
\sum_{0 \leq k \leq m}(-1)^{m-k}\left(\begin{array}{c}
m \\
k
\end{array}\right) a_{r k+j}=0 \quad \text { for all } j \geq 0 .
$$

Since $T^{s}$ is a $n$-quasi strict $l$-isometry, it follows that

$$
\sum_{0 \leq k \leq l}(-1)^{l-k}\left(\begin{array}{l}
l \\
k
\end{array}\right)\left\|T^{s(n+k)} x\right\|^{2}=0=\sum_{0 \leq k \leq l}(-1)^{l-k}\left(\begin{array}{l}
l \\
k
\end{array}\right)\left\|T^{s(n+k)+n(r-s)} x\right\|^{2} .
$$

Then $\left(a_{s j}\right)_{j \geq 0}$ is an arithmetic progression of strict order $l-1$ satisfies the recursive equation

$$
\sum_{0 \leq k \leq l}(-1)^{l-k}\left(\begin{array}{l}
l \\
k
\end{array}\right) a_{s k+j}=0 \quad \text { for all } j \geq 0 .
$$

Applying Lemma 3.8 it results that $\left(a_{q j}\right)_{j \geq 0}$ is an arithmetic progression of strict order $p-1$, so $T^{q}$ is an $n$-quasi-strict $p$-isometry, where $q=\operatorname{pgcd}(r, s)$ and $p=\min \{m, l\}$.

The following corollary shows that if two suitable different powers of $T$ are $n$-quasi strict- $m$-isometries, then $T$ is a $n$-quasi strict- $m$-isometry. 
Corollary 3.10. Let $T \in \mathcal{L}(\mathcal{H})$ and $r, s, m, n, l$ be positive integers. The following properties hold.

(1) If $T$ is an $n$-quasi strict-m-isometry such that $T^{s}$ is an $n$-quasi strict-isometry, then $T$ is an $n$-quasi strict-isometry.

(2) If $T^{r}$ and $T^{r+1}$ are $n$-quasi strict-m-isometries, then so is $T$.

(3) If $T^{r}$ is an n-quasi strict-m-isometry and $T^{r+1}$ is an n-quasi strict-l-isometry with $m<l$, then $T$ is an $n$-quasi strict-m-isometry.

Proof. The proof is an immediate consequence of Theorem 3.9.

Theorem 3.11. Let $T \in \mathcal{L}(\mathcal{H})$ and $S \in \mathcal{L}(\mathcal{H})$ be doubly commuting operators. If $T$ is an $n$-quasi strict $m$-isometry and $S$ is an n-quasi strict l-isometry, then $T S$ is an $n$-quasi strict $(m+l-1)$-isometry if and only if

$$
\left(T^{*}\right)^{n+l-1} \beta_{m-1}(T) T^{n+l-1} S^{* n} \beta_{l-1}(S) S^{n}=0 .
$$

Proof. In view of Theorem 2.18, it is obvious that $\beta_{m+l-1, n}(T S)=0$. On the other hand, since $T$ and $S$ are doubly commuting operators, it follows from [14, Corollary 3.9] that

$$
\beta_{q}(T S)=\sum_{0 \leq k \leq q}\left(\begin{array}{l}
q \\
k
\end{array}\right) T^{* k} \beta_{q-k}(T) T^{k} \beta_{k}(S),
$$

from which it follows that

$$
\begin{aligned}
& \beta_{m+l-2, n}(T S) \\
&=(T S)^{* n} \beta_{m+l-2}(T S)(T S)^{n} \\
&=\left(T^{*}\right)^{n}\left(S^{*}\right)^{n}\left(\sum_{0 \leq k \leq m+l-2}\left(\begin{array}{c}
m+l-2 \\
k
\end{array}\right) T^{* k} \beta_{m+l-2-k}(T) T^{k} \beta_{k}(S)\right) T^{n} S^{n} \\
&= \sum_{0 \leq k \leq m+l-2}\left(\begin{array}{c}
m+l-2 \\
k
\end{array}\right) T^{* k}\left(T^{*}\right)^{n} \beta_{m+l-2-k}(T) T^{n} T^{k}\left(S^{*}\right)^{n} \beta_{k}(S) S^{n} \\
&= \sum_{0 \leq k \leq l-2}\left(\begin{array}{c}
m+l-2 \\
k
\end{array}\right) T^{* k}\left(T^{*}\right)^{n} \beta_{m+l-2-k}(T) T^{n} T^{k}\left(S^{*}\right)^{n} \beta_{k}(S) S^{n} \\
&+\left(\begin{array}{c}
m+l-2 \\
l-1
\end{array}\right)\left(T^{*}\right)^{n+l-l} \beta_{m-1}(T) T^{n+l-1}\left(S^{*}\right)^{n} \beta_{l-1}(S) S^{n} \\
&+\sum_{l \leq k \leq m+l-2}\left(\begin{array}{c}
m+l-2 \\
k
\end{array}\right) T^{* k}\left(T^{*}\right)^{n} \beta_{m+l-2-k}(T) T^{n} T^{k}\left(S^{*}\right)^{n} \beta_{k}(S) S^{n} .
\end{aligned}
$$

If $k \in[0, l-2]$, then $(m+l-2-k) \in[m, m+l-2]$ and hence $T^{* n} \beta_{m+l-2-k}(T) T^{n}=0$ by Corollary 2.7. If $k \geq l$, then $S^{n} \beta_{k}(S) S^{n}=0$ also in view of Corollary 2.7.

Consequently, $T S$ is a $n$-quasi strict- $(m+l-1)$-isometry if and only if

$$
\left(T^{*}\right)^{n+l-1} \beta_{m-1}(T) T^{n+l-1}\left(S^{*}\right)^{n} \beta_{l-1}(S) S^{n}=0 .
$$

Hence the proof is finished.

Theorem 3.12. Let $T \in \mathcal{L}(\mathcal{H})$ and $S \in \mathcal{L}(\mathcal{H})$. If $T$ is an n-quasi strict-m-isometry and $S$ is an n-quasi strict-l-isometry, then $T \otimes S$ on $\mathcal{H} \bar{\otimes} \mathcal{H}$ is an n-quasi strict- $(m+l-1)$ isometry. 
Proof. In view of [12, Corollary 3.10], it follows that

$$
\beta_{q}(T \otimes S)=\sum_{0 \leq k \leq q}\left(\begin{array}{l}
q \\
k
\end{array}\right) T^{* k} \beta_{q-k}(T) T^{k} \otimes \beta_{k}(S) .
$$

By calculations we have

$$
\begin{aligned}
& \beta_{m+l-2, n}(T \otimes S) \\
= & (T \otimes S)^{* n} \beta_{m+l-2}(T \otimes S)(T \otimes S)^{n} \\
= & \left(T^{* n} \otimes S^{* n}\right)\left(\sum_{0 \leq k \leq m+l-2}\left(\begin{array}{c}
m+l-2 \\
k
\end{array}\right) T^{* k} \beta_{m+l-2-k}(T) T^{k} \otimes \beta_{k}(S)\right)\left(T^{n} \otimes S^{n}\right) \\
= & \sum_{0 \leq k \leq m+l-2}\left(\begin{array}{c}
m+l-2 \\
k
\end{array}\right) T^{* k} T^{* n} \beta_{m+l-2-k}(T) T^{n} T^{k} \otimes S^{* n} \beta_{k}(S) S^{n} .
\end{aligned}
$$

Similar arguments as in the proof of Theorem 3.11 give

$$
\beta_{m+l-2, n}(T \otimes S)=\left(T^{*}\right)^{n+l-1} \beta_{m-1}(T) T^{n+l-1} \otimes\left(S^{*}\right)^{n} \beta_{l-1}(S) S^{n} .
$$

This means that $T \otimes S$ is a $n$-quasi strict $(m+l-1)$-isometry as required.

In [7, Theorem 3.1] it has been proved that if $T \in \mathcal{L}(\mathcal{H})$ is a strict $m$-isometry, then the list of operators $\left\{T^{* k} T^{k}, k=0,1, \cdots m-1\right\}$ is linearly independent which is equivalent to the fact that $\left\{\beta_{k}(T), k=0,1, \cdots, m-1\right\}$ is linearly independent.

In the following proposition we extend this result to $n$-quasi strict $m$-isometries as follows.

Proposition 3.13. If $T \in \mathcal{L}(\mathcal{H})$ is an n-quasi strict $m$-isometry, then the list of operators

$$
\left\{\beta_{k, n}(T), k=0,1, \cdots, m-1\right\}
$$

is linearly independent.

Proof. The outline of the proof is inspired from [10].

It was observed in [12] that $\beta_{k}(T)=T^{*} \beta_{k-1}(T) T-\beta_{k-1}(T)$ for all $k \geq 1$.

We will just write

$$
\begin{aligned}
\beta_{m, n}(T)=T^{* n} \beta_{m}(T) T^{n} & =T^{* n}\left(T^{*} \beta_{m-1}(T) T-\beta_{m-1}(T)\right) T^{n} \\
& =T^{* n+1} \beta_{m-1}(T) T^{n+1}-T^{* n} \beta_{m-1}(T) T^{n} .
\end{aligned}
$$

Now assume that for some complex numbers $\lambda_{k}$,

or equivalently

$$
\sum_{0 \leq k \leq m-1} \lambda_{k} \beta_{k, n}(T)=0
$$

$$
\sum_{0 \leq k \leq m-1} \lambda_{k} T^{* n} \beta_{k}(T) T^{n}=0 .
$$

Multiplying the above equation on the left and right by $T^{*}$ and $T$ and subtracting two equations, we have

$$
\sum_{0 \leq k \leq m-1} \lambda_{k}\left(T^{* n+1} \beta_{k}(T) T^{n+1}-T^{* n} \beta_{k}(T) T^{n}\right)=\sum_{0 \leq k \leq m-1} \lambda_{k} \beta_{k+1, n}(T)=0 .
$$

By applying the same procedure to the equation $\sum_{0 \leq k \leq m-1} \lambda_{k} \beta_{k+1, n}(T)=0$ we get

$$
\sum_{0 \leq k \leq m-1} \lambda_{k} \beta_{k+2, n}(T)=0
$$


By continuing this process we obtain

$$
\sum_{0 \leq k \leq m-1} \lambda_{k} \beta_{k+j, n}(T)=0 \text { for all } j \in \mathbb{N} .
$$

Since every $n$-quasi- $m$-isometric operator is an $n$-quasi- $k$-isometric operator for all $k \geq m$ (Corollary 2.7 ) we have the following cases:

For $j=m-1, \sum_{0 \leq k \leq m-1} \lambda_{k} \beta_{k+j, n}(T)=0 \Rightarrow \lambda_{0} \beta_{m-1, n}(T)=0$, so $\lambda_{0}=0$.

For $j=m-2, \sum_{0 \leq k \leq m-1} \lambda_{k} \beta_{k+j, n}(T)=0 \Rightarrow \lambda_{1} \beta_{m-1, n}(T)=0$, so $\lambda_{1}=0$.

Continuing this process we see that all $\lambda_{k}=0$ for $k=2, \cdots, m-1$. Hence the result is proved.

Acknowledgment. The authors wish to thank the referee for suggestions which led to a significant improvement of this paper.

\section{References}

[1] J. Agler and M. Stankus, m-isometric transformations of Hilbert space. I, Integral Equations Operator Theory 21, 383-429, 1995.

[2] T. Bermúdez, A. Martinón, V. Müller and J. A. Noda, Perturbation of m-Isometries by Nilpotent Operators, Abstr. Appl. Anal. 2014, Article ID 745479, 6 pages, 2014.

[3] T. Bermúdez, A. Martinón and J.A. Noda, Products of m-isometries, Linear Algebra Appl. 438, 80-86, 2013.

[4] T. Bermúdez, A. Martinón and J.A. Noda, An isometry plus a nilpotent operator is an m-isometry. Applications, J. Math. Anal. Appl. 407 (2), 505-512, 2013.

[5] T. Bermúdez, A. Martinón and J.A. Noda, Arithmetic Progressions and Its Applications to $(m, q)$-Isometries: A Survey. Results Math. 69, 177-199, 2016.

[6] T. Bermúdez, C.D. Mendoza and A. Martinón, Powers of m-isometries, Studia Math. 208 (3), 2012.

[7] F. Botelho, J. Jamison and B. Zheng, Strict isometries of arbitrary orders, Linear Algebra Appl. 436, 3303-3314, 2012.

[8] M. Chō, S. Óta and K. Tanahashi, Invertible weighted shift operators which are $m$ isometries, Proc. Amer. Math. Soc. 141 (12), 4241-4247, 2013.

[9] B.P. Duggal, Tensor product of n-isometries, Linear Algebra Appl. 437, 307-318, 2012.

[10] C. Gu, Elementary operators which are m-isometries, Linear Algebra Appl. 451, 49-64, 2014.

[11] C. Gu, Structures of left n-invertible operators and their applications, Studia Math. 226 (3), 189-211, 2015.

[12] C. Gu, Functional calculus for $m$-isometries and related operators on Hilbert spaces and Banach spaces, Acta Sci. Math. (Szeged) 81, 605-641, 2015.

[13] C. Gu, Examples of m-isometric tuples of operators on a Hilbert space, J. Korean Math. Soc. 55 (1), 225-251, 2018.

[14] C. Gu and M. Stankus, Some results on higher order isometries and symmetries: Products and sums with a nilpotent operator, Linear Algebra Appl. 469, 500-509, 2015.

[15] J. Kyu Han, H. Youl Lee and W. Young Lee, Invertible completions of $2 \times 2$ upper triangular operator matrices, Proc. Amer. Math. Soc. 128, 119-123, 1999.

[16] O.A. Mahmoud Sid Ahmed, m-isometric operators on Banach spaces, AsianEuropean J. Math. 3 (1), 19 pages, 2010. 
[17] O.A. Mahmoud Sid Ahmed, Generalization of m-partial isometries on a Hilbert spaces, Int. J. Pure Appl. Math. 104 (4), 599-619, 2015.

[18] S. Mecheri and S. M. Patel, On quasi-2-isometric operators, Linear Multlinear Algebra 66 (5), 1019-1025, 2018.

[19] S. Mecheri and T. Prasad, On n-quasi-m-isometric operators, Asian-Eur. J. Math. 9 (3), 1650073, 8 pages, 2016.

[20] S.M. Patel, A note on quasi-isometries, Glas. Mat. 35 (55), 307-312, 2002.

[21] S.M. Patel, 2-isometric operators, Glas. Mat. 37 (57), 141-145, 2002.

[22] S.M. Patel, A note on quasi-isometries II, Glas. Mat. 38 (58), 111-120, 2003.

[23] M.A. Rosenblum, On the operator equation $B X-X A=Q$, Duke Math. J. 23, 263-269, 1956.

[24] A. Saddi and O. A. Mahmoud Sid Ahmed, m-partial isometries on Hilbert, spaces Intern. J. Funct. Anal. Operators Theory Appl. 2 (1), 67-83, 2010.

[25] L. Suciu, Quasi-isometries in semi-Hilbertian spaces, Linear Algebra Appl. 430, 24742487, 2009 . 